\title{
Impulse Propagation along Thalamocortical Fibers Can Be Detected Magnetically outside the Human Brain
}

\author{
Tomoaki Kimura, ${ }^{1}$ Isamu Ozaki, ${ }^{2}$ and Isao Hashimoto ${ }^{3}$ \\ ${ }^{1}$ Tsukuba University of Technology, Tsukuba 305-8521, Japan, ${ }^{2}$ Aomori University of Health and Welfare, Aomori 030-8505, Japan, and ${ }^{3}$ Kanazawa \\ Institute of Technology, Tokyo Office, Tokyo 102-0083, Japan
}

\begin{abstract}
Orchestrating cortical network activity with synchronous oscillations of neurons across distant regions of the brain underlies information processing in humans (Knight, 2007) and monkeys (Saalmann et al., 2007; Womelsdorf et al., 2007). Frequencies of oscillatory activities depend, to a considerable extent, on the length and conduction velocity of the tracts connecting the neural areas that participate in oscillations (Buzsáki, 2006). However, the impulse propagation along the fiber tracts in the white matter has never been visualized in humans. Here, we show, by recording magnetoencephalogram (MEG) following median nerve stimulation, that a magnetic field component, we labeled "M15," changes dynamically within 1.6-1.8 ms before the onset of magnetic M20 response generated from the primary somatosensory cortex. This new M15 component corresponds to the intracellular depolarizing action current in the thalamocortical fibers propagating with the mean conduction velocity of $29 \mathrm{~m} / \mathrm{s}$. The findings challenge the traditional view that MEG is blind to the activity of deep subcortical structures. We argue that the MEG technique holds the promise of providing novel information in impulse transmissions along not only the thalamocortical pathway but also other fiber tracts connecting distant brain areas in humans.
\end{abstract}

Key words: human thalamocortical fibers; impulse propagation; conduction velocity; magnetoencephalography; somatosensory evoked fields; M15 component

\section{Introduction}

External stimuli such as visual or auditory are decomposed into distinctive features and are processed in different parts of the brain. Synchronous activation of neurons in different regions underlies various stages of information processing (Knight, 2007). In this context, detecting oscillatory activity of the brain offers new insights in the functioning human brain. In general, slow oscillations involve long-range networks, whereas fast oscillations involve short-range networks, i.e., the distance between the recruited neuronal pools is inversely related to their frequency (Buzsáki, 2006). For better understanding coactivation of discrete cortical regions, we need to know firstly reciprocal propagations of the impulses along the fiber tracts connecting those regions and secondly the frequencies of concurrent neural activations.

Whereas magnetic recordings have demonstrated impulse propagations along the peripheral nerve as moving sources of a dipole or a quadrupole of compound action fields (CAFs) (Wikswo et al., 1980; Hari et al., 1989; Curio et al., 1991; Hashimoto et al., 1994), magnetoencephalography (MEG) is considered to be only sensitive to postsynaptic potentials in pyramidal neurons of the cerebral cortex that are lined-up along mainly tangential orientation. The common belief is that MEG is blind to deep and

Received June 1, 2008; revised Sept. 19, 2008; accepted 0ct. 16, 2008.

This work was supported by Ishikawa High-Tech Sensing Cluster, whom we thank. We thank Yasuyuki Kawabuchi for technical assistance.

Correspondence should be addressed to Isao Hashimoto, Kanazawa Institute of Technology, IshikawakenKioikaikan, Room 204, 4-8 Kojimachi, Chiyoda-ku, Tokyo 102-0083, Japan. E-mail: ihashi@ael.kanazawa-it.ac.jp. D0I:10.1523/JNEUROSCI.3022-08.2008

Copyright $\odot 2008$ Society for Neuroscience $\quad 0270-6474 / 08 / 2812535-04 \$ 15.00 / 0$ radial sources owing to the cancellation by the magnetic field of the secondary volume currents. Although a theoretical simulation showed that the sensitivity profile of MEG does not exclude deep sources (Fagaly, 2006), no previous studies on MEG have disclosed impulse propagations within the white matter in the human brain. In the present study, we visualized using MEG impulse propagations along the fibers from the thalamus to the somatosensory cortex and determined the conduction velocity of thalamocortical volleys.

\section{Materials and Methods}

Three healthy paid volunteers (male adults, 23-25 years old, 2 righthanded) participated in the study. All subjects gave their written informed consent before the experiment. Using a 160-channel whole-head type axial gradiometer system (MEGvision PQ1160C, Yokogawa Electric), we recorded MEG (low-pass frequency: $2000 \mathrm{~Hz}$ ) during wakefulness in a magnetically shielded room (Daido Steel). The shielding factor was $\sim 85 \mathrm{~dB}$ at $1 \mathrm{~Hz}$ and $100 \mathrm{~dB}$ at $10 \mathrm{~Hz}$ and above. Sensors are configured as first-order axial gradiometers with a baseline of $50 \mathrm{~mm}$; each coil of the gradiometers measures $15.5 \mathrm{~mm}$ in diameter The sensors are arranged in a uniform array on a helmet-shaped surface at the bottom of the dewar and the mean distance between the centers of two adjacent coils is $25 \mathrm{~mm}$. The field sensitivities of the sensors (the noise of the system) were $3 \mathrm{fT} / \sqrt{ } \mathrm{Hz}$ at the frequency range of M15 between 50 and $300 \mathrm{~Hz}$ (see Results).

Constant current pulses (rectangular shape, $0.2 \mathrm{~ms}$ duration) were delivered to the left median nerve at the wrist at irregular intervals with interstimulus intervals between 250 and $750 \mathrm{~ms}$. The stimulus intensity was three times sensory threshold, eliciting a mild twitch of the abductor pollicis brevis muscle. To obtain somatosensory evoked magnetic fields (SEF), an epoch of $150 \mathrm{~ms}$ duration (50 ms pre and $100 \mathrm{~ms}$ poststimulus) was digitized at a $10 \mathrm{kHz} /$ channel sampling rate and 2500 responses were 
averaged on-line. The wide-band recorded responses were digitally filtered with a bandpass of 5-300 Hz. When analyzing each SEF response, we corrected baselines for all recorded channels by DC offset based on the period between 5 and $10 \mathrm{~ms}$ after stimulus onset where no brain signals were present. We also evaluated the noise level of the SEF response by calculating (system noise level $) \times(\sqrt{ }$ (frequency of low-pass filter $) /$ $\checkmark$ (number of average) (Hari et al., 1988; Hashimoto et al., 1996).

We attached coils at 5 locations of the head surface as the fiduciary points with respect to the landmarks (nasion and preauricular points). For each recording session, we determined the position of the head within the helmet by measuring the magnetic fields after passing currents through the coils. A 3D head coordinate system was applied to express the location and orientation of equivalent current dipoles (ECDs). The $x$-axis pointed from the origin of $3 \mathrm{D}$ head coordinate system (the midpoint of the preauricular points) to the left preauricular point, the $y$-axis to the direction opposite from the nasion, and the $z$-axis to the vertex in a direction perpendicular to the X-Y plane.

Using Signa Excite HD 1.5 T system (GE Yokogawa), all subjects underwent T1-weighted magnetic resonance imaging (MRI) study with spherical lipid markers placed at the 5 MEG fiduciary points that enabled us to superpose the MEG coordinate system on the MRI. The MRI consisted of 166 sequential slices of $1.2 \mathrm{~mm}$ thickness, with a resolution of $512 \times 512$ points in a field of view of $261 \times 261 \mathrm{~mm}$. After reconstructing three dimensional MRI, the best-fit sphere was determined for each subject's head.

We used a single ECD model in a spherical volume conductor for identifying sources of the magnetic signals. We calculated dipole strength, location and orientation of the ECDs for the SEF response between 12 and $22 \mathrm{~ms}$ poststimulus using the data from 80 to 100 of 160 channels that cover both entering and leaving magnetic flux. We accepted ECDs with a goodness-of-fit (GOF) value of $>60 \%$ and superimposed accepted ECDs onto each individual subject's MRI to confirm those anatomical locations. We normalized dynamic movement of ECDs for the three subjects for $x-, y$-, and $z$-axis from the mean location of the ECDs at the peak latency of M20.

\section{Results}

Figure 1 illustrates the results of SEF following left median nerve stimulation that were obtained from a representative subject. In this study, we focused on the small magnetic deflection (M15) before the initial cortical M20 component. This M15 culminated at $14.8 \mathrm{~ms}$ (Fig. $1 \mathrm{Ba}$ ) and continued to the onset of M20 at 16.7 ms (Fig. $1 B c$ ). The mean maximum signal strength of the M15 was 15.7 femto-tesla (fT), 11 times larger than the noise level of $1.3 \mathrm{fT}$ measured from the baseline period. This value is close to the theoretically calculated noise level of $3 \mathrm{fT} \times \sqrt{ } 300 / \sqrt{ } 2500=$ 1.03 fT (Hari et al., 1988; Hashimoto et al., 1996). Figure $1 C$ shows isocontour field distributions of the magnetic fields for the M15 and M20 components. A long distance between the maximum flux-out and minimum flux-in for M15 component over the head surface, as well as wide intervals between any adjacent contour lines for flux-out or flux-in (Fig. $1 C a, b$ ) reflects deep current sources for the M15. These findings present a striking contrast with those of the M20 located superficially at the somatosensory cortex showing a magnetic field pattern with the short distance between the maximum flux-out and minimum flux-in and narrow intervals between the adjacent contour lines (Fig. $1 C c$ and asterisk).

We estimated the location and orientation of ECDs successively from the M15 onset to the M20 peak. The ECD at M20 peak latency was located at the hand area of right somatosensory cortex, shown as an asterisk in the top panel of Figure $1 D$. Figure $1 D$ illustrates changes in the location of ECDs for the M15-M20 continuum with color-gradation from yellow to purple that corresponds to the time courses in Figure 1, $A$ and $B$. The orientation of the ECD varied along the time course of the M15 component; from time (Fig. $1 \mathrm{Ba}$ ) to time (Fig. $1 \mathrm{Bb}$ ), the orientation of M15
ECD was upward. From time (Fig. $1 B c$ ) to time (Fig. $1 A$, asterisk), the ECD changed its orientation to the front and perpendicular to the central sulcus. This is identical with the orientation of the M20 ECD. Here, the time (Fig. $1 B c$ ) was judged as the onset of the M20 component so that an S-shaped colored curve in the coronal or a C-shaped curve in the sagittal view of MRI (Fig. 1D) indicates sequential changes in ECD location from the thalamus (yellow) to primary somatosensory cortex (purple), demonstrating upward impulse propagation along the thalamocortical fibers.

Figure 2 summarizes the results of ECD analysis across three subjects. The mean onset latency $(16.4 \pm 0.3 \mathrm{~ms})$ of the M20 component is adjusted to time 0 (Fig. $2 \mathrm{Ac}$ ). The GOF values were above $75 \%$ for the M15 ECDs in two subjects and above $60 \%$ in one subject. At (Fig. $2 \mathrm{Aa}$ ), $1.8 \mathrm{~ms}$ before the M20 onset, the mean ECD location was $-17.5 \pm 0.2$ (mean \pm SD) $\mathrm{mm}$ for X-, $-8.9 \pm$ $0.6 \mathrm{~mm}$ for $\mathrm{Y}$ - and $55.5 \pm 8.5 \mathrm{~mm}$ for $z$-axis near the thalamus. During the $1.8 \mathrm{~ms}$-period from time (Fig. 2Aa) to (Fig. 2Ac), the ECD moved upward, anteriorly and laterally in the direction of the right central scalp. At time (Fig. $2 A c$ ), the onset latency of the $\mathrm{M} 20$, the mean ECD location was $-39.0 \pm 3.7 \mathrm{~mm}$ for X-, $-17.2 \pm 10.0 \mathrm{~mm}$ for $\mathrm{Y}$ - and $92.9 \pm 7.1 \mathrm{~mm}$ for $z$-axis. The mean Euclidian distance of the M15 ECD movement from time (Fig. $2 A a$ ) to (Fig. $2 A c$ ) during the period of $1.8 \mathrm{~ms}$ was $48.8 \mathrm{~mm}$ (range, $44.5-56.5 \mathrm{~mm}$ ). Thus, the conduction velocity as reflected in M15 ECD movement was estimated at $29 \mathrm{~m} / \mathrm{s}$ (range, 25-35 m/s). As shown in Figure $2 B$, the ECD remained stable after the M20 onset. At the peak latency of M20 (20.0 $\pm 0.6 \mathrm{~ms})$, the mean ECD location was $-49.7 \pm 1.5 \mathrm{~mm}$ for X-, $-20.4 \pm 9.7$ $\mathrm{mm}$ for $\mathrm{Y}$ - and $95.1 \pm 2.4 \mathrm{~mm}$ for $z$-axis, coinciding with the $3 \mathrm{~b}$ hand area of somatosensory cortex. The mean maximal dipole strength of the M15 across three subjects was 10.4 (range, 3.714.4) nAm and that of the M20, 22.1 (range, 11.1-34.5) nAm.

\section{Discussion}

In SEFs following median nerve stimulation, we found a new magnetic component "M15" culminating at 1.6-1.8 ms before the M20 onset. The M15 magnetic field over the head surface dynamically changes during the short period: from the field pattern suggestive of a deep current source for the initial part of M15 to that of a superficial current source for the last part of M15. The estimated ECD of the initial part of M15, superimposed on to the subject MRI, was located in the thalamus. The ECD of the last part of M15 was located near the hand area of the primary somatosensory cortex. During the whole period of M15, the ECD successively moves from the thalamus to the hand area of the somatosensory cortex in the postcentral gyrus with the S-shaped track in the MRI coronal view that corresponds to the thalamocortical pathway. We therefore consider that the M15 current source, directing upward in an initial stage, reflects the leading dipole, the longitudinal intracellular depolarization current, in the thalamocortical fibers. In support of this conjecture, we have shown in the human peripheral nervous system that the magnitude of depolarization field of CAFs was larger than that of repolarization field by 3 times or more (Hashimoto et al., 1994). Extrapolating from the peripheral nerve into the cerebral white matter, we speculate that only the depolarization field of the quadrupolar pattern of the CAFs along the thalamocortical pathway was detected by the sensors located remote from the signal source in the present study. The single ECD estimation of the quadrupole and the long distance between the sources and detection coils may render the GOF of M15 ECDs lower than that of cortical M20 ECDs.

Our findings challenge the prevailing view that MEG is blind 

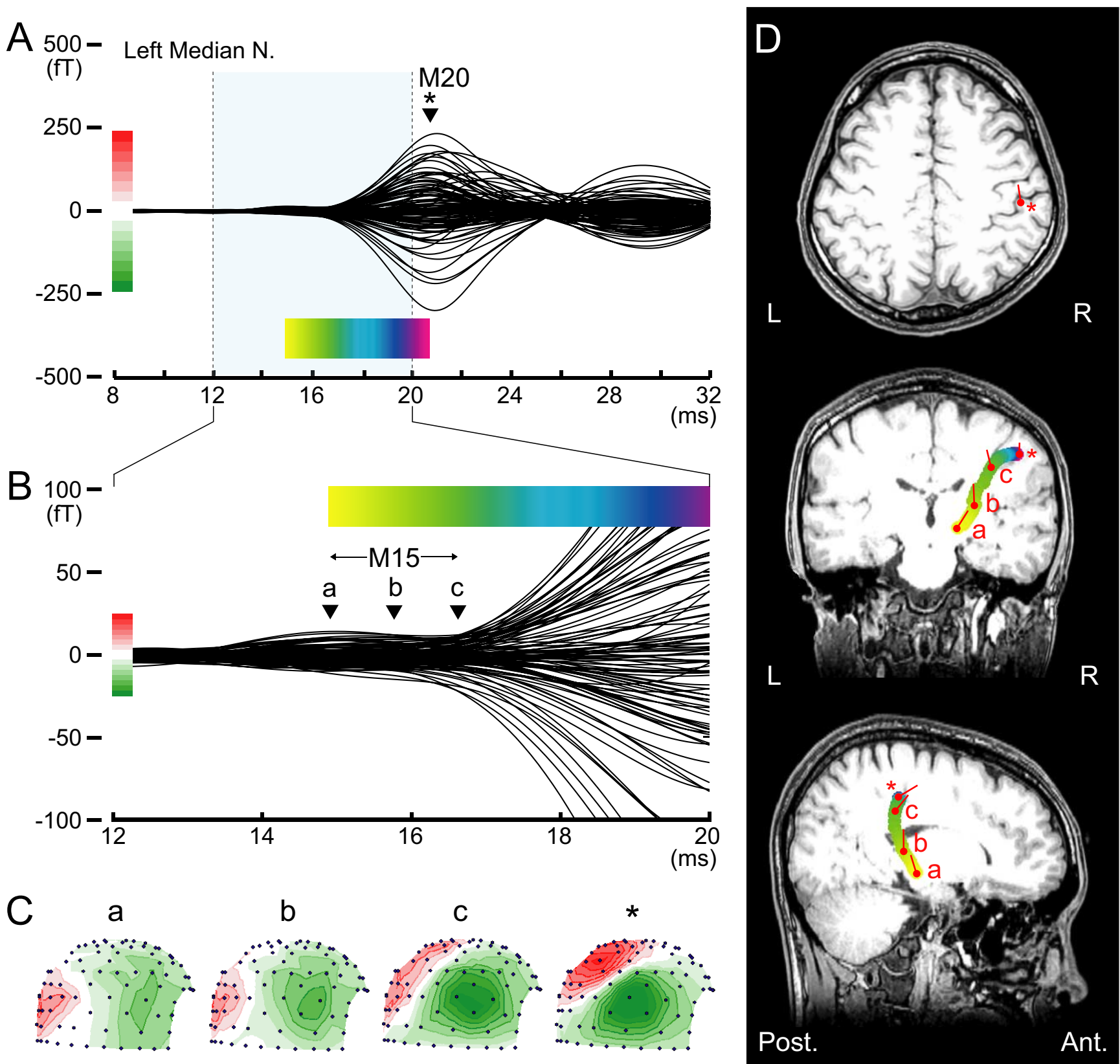

Figure 1. Somatosensory magnetic fields following left median nerve stimulation in a 23 -year-old man. $A$, Superimposed waveforms recorded from the right hemisphere ( 80 locations) show a clear M20 magnetic deflection; at $20.6 \mathrm{~ms}\left(^{*}\right)$ after stimulus onset, the ECD is estimated in hand area of the postcentral gyrus on the right side (D, top). $\boldsymbol{B}$, M15 deflection before M20 onset, lasting from 14.9 (a) to 16.7 (c) ms poststimulus, is highlighted with expanded scales for both magnetic strength and time. $\boldsymbol{C}$, Right lateral view of isocontour field distributions of the magnetic field over the head surface at 4 time points; red contours, magnetic flux-out; green contours, flux-in with contour step of $3 \mathrm{fT}$ for the latency of $\boldsymbol{a}-\boldsymbol{c}$ in $\boldsymbol{B}$ and $30 \mathrm{ft}$ for M20 peak (asterisk) in $\boldsymbol{A}$. Note that the long distance between the maximum flux-out and minimum flux-in and wide intervals between any adjacent contour lines for flux-out or flux-in ( $\boldsymbol{a}$ and $\boldsymbol{b}$ ) suggest deep current sources. In contrast, the short distance between the maximum flux-out and minimum flux-in and narrow intervals between the adjacent contour lines (c and asterisk) indicate superficial current sources. $\boldsymbol{D}$, Estimated ECDs superimposed onto the subject MRI (from top; horizontal, coronal and sagittal views). Changes in locations of the ECDs calculated successively at a 0.1 ms step until the M20 peak are illustrated as a colored curve, in which color gradation from yellow to purple corresponds to the time course of the colored bar in $\boldsymbol{A}$ or $\boldsymbol{B}$. Note that $S$-shaped colored curve in the coronal view indicates sequential changes in ECD location from thalamus (yellow) to primary somatosensory cortex (purple), demonstrating impulse propagation along the thalamocortical fibers. Red circles and bars indicate the location and orientation of ECD for the latency of $\boldsymbol{a}-\boldsymbol{c}$ in $\boldsymbol{B}$ or an asterisk in $\boldsymbol{A}$.

to activity of deep subcortical structures. We have shown that both localization and tracking of a moving source in the deep brain is feasible with high spatial as well as temporal resolution with first order axial SQUID gradiometers. With sufficient signal-to-noise ratio of responses and an appropriate experimental design, it is possible to record MEG from deep regions. This is in accordance with a previous report on MEG that demonstrated time-locked activities from the cerebellum ipsilateral to the site of median nerve stimulation (Hashimoto et al., 2003).
Theoretically, first order axial gradiometers with a baseline of $50 \mathrm{~mm}$ used in the present study can record magnetic signals produced by the current source that is $100 \mathrm{~mm}$ distant from the detection coils, with a fall off of less than half of its signal power (Fagaly, 2006) and the thalamocortical pathway is located within this distance. By evaluating dynamic movement of the M15 source, we demonstrated that the mean conduction velocity of the thalamocortical fibers is $29 \mathrm{~m} / \mathrm{s}$ in humans. This value is in accordance with a predicted value of $33 \mathrm{~m} / \mathrm{s}$ based on the diam- 
eters of axons from the specific thalamic nucleus and on the latency analysis of somatosensory evoked potentials in humans (Desmedt and Cheron, 1980).

Our success to record magnetic signals produced by propagating action potentials in the brain contradicts the common belief that MEG is only sensitive to postsynaptic potentials (EPSPs/IPSPs) of a particular class of neurons. In general, action potentials propagating along the nerve fibers in deep brain have been considered undetectable by MEG measurement, since magnetic fields produced by the primary intracellular currents are cancelled by those produced by the secondary extracellular return currents that are widely distributed within the head. In other words, the visibility of deep sources in MEG strongly depends on the amount of cancellation between the field of the primary and secondary currents. Therefore, for deep sources, we have to use an accurate volume conductor model taking into account the deviation of the head from a sphere. However, we argue that a single ECD model in a spherical volume conductor is appropriate in the analysis of SEF. Because local curvature of the central scalp can be well approximated by a spherical surface and the deviation of individual subject's head from a sphere is negligible when analyzing current sources of cortical or subcortical structures beneath the central scalp. However, for deep as well as superficial sources in the frontal or occipital areas, it is preferable to use more accurate volume conductor models, such as a real head shape model or a multisphere model in which for each sensor a sphere is fitted to the local curvature, thereby minimizing the deviation of the head from a sphere (Huang et al. 1999).

Contrary to the traditional notion, the current study has demonstrated that the MEG enables us to record propagating action potentials from the thalamus to the somatosensory cortex. We conclude that the MEG has the capability of providing novel information in functioning human brain by delineating impulse transmissions along the fiber tracts connecting distant brain areas noninvasively.

\section{References}

Buzsáki G (2006) Rhythms of the brain. New York, Oxford UP.

Curio G, Erné SN, Sandfort J, Scheer J, Stehr R, Trahms L (1991) Exploratory mapping of evoked neuromagnetic activity from human peripheral nerve, brachial plexus and spinal cord. Electroencephalogr Clin Neurophysiol 81:450-453.

Desmedt JE, Cheron G (1980) Central somatosensory conduction in man: neural generators and interpeak latencies of the far-field components recorded from neck and right or left scalp and earlobes. Electroencephalogr Clin Neurophysiol 50:382-403.

Fagaly RL (2006) Superconducting quantum interference device instruments and applications. Rev Sci Instrum 77:101101-1-101101-45.

Hari R, Joutsiniemi SL, Sarvas J (1988) Spatial resolution of neuromagnetic records: theoretical calculations in a spherical model. Electroencephalogr Clin Neurophysiol 71:64-72.
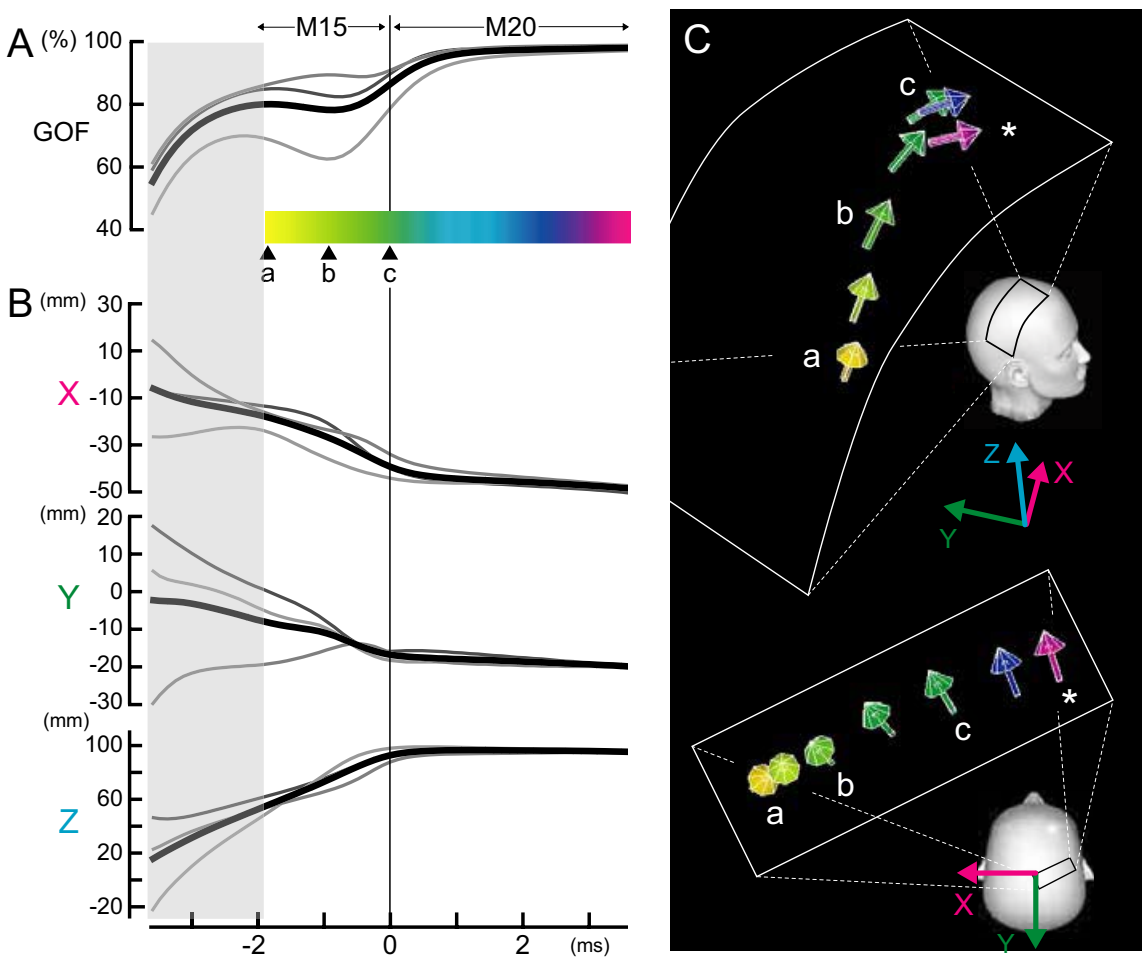

Figure 2. Changes in locations and orientations of the ECDs for M15-M20 in three subjects. $A$, Gray lines represent the GOF values of M15 ECDs in each subject and the black line, the average across three subjects. The GOF values were above $75 \%$ for two and above $60 \%$ for one subject. A horizontal colored bar with color gradation from yellow to purple corresponds to the

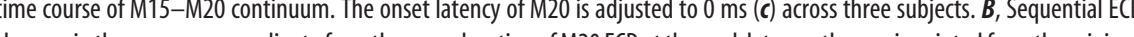
a color gradation from yellow to purple corresponds to the time course of M15-M20 continuum (asterisk, M20 peak). Note that the averaged ECD for M15 shows changes in orientation during the period between $\boldsymbol{a}$ and $c$, whereas that for M20 (blue or purple arrow) is horizontally oriented toward midfrontal scalp.

Hari R, Hällström J, Tiihonen J, Joutsiniemi SL (1989) Multichannel detection of magnetic compound action fields of median and ulnar nerves. Electroencephalogr Clin Neurophysiol 72:277-280.

Hashimoto I, Mashiko T, Mizuta T, Imada T, Iwase K, Okazaki H (1994) Visualization of a moving quadrupole with magnetic measurements of peripheral nerve action fields. Electroencephalogr Clin Neurophysiol 93:459-467.

Hashimoto I, Mashiko T, Imada T (1996) Somatic evoked high-frequency magnetic oscillations reflect activity of inhibitory interneurons in the human somatosensory cortex. Electroencephalogr Clin Neurophysiol 100:189-203.

Hashimoto I, Kimura T, Tanosaki M, Iguchi Y, Sekihara K (2003) Muscle afferent inputs from the hand activate human cerebellum sequentially through parallel and climbing fiber systems. Clin Neurophysiol 114:2107-2117.

Huang MX, Mosher JC, Leahy RM (1999) A sensor-weighted overlappingsphere head model and exhaustive head model comparison for MEG. Phys Med Biol 44:423-440.

Knight RT (2007) Neural networks debunk phrenology. Science 316:1578-1579.

Saalmann YB, Pigarev IN, Vidyasagar TR (2007) Neural mechanisms of visual attention: how top-down feedback highlights relevant locations. Science 316:1612-1615.

Wikswo JP, Barach JP, Freeman JA (1980) Magnetic field of a nerve impulse: first measurements. Science 208:53-55.

Womelsdorf T, Schoffelen JM, Oostenveld R, Singer W, Desimone R, Engel AK, Fries $P$ (2007) Modulation of neuronal interactions through neuronal synchronization. Science 316:1609-1612. 